PRIMERAS JORNADAS CIENTÍFICAS DE ESTUDIANTES DE ENFERMERÍA. FACULTAD CIENCIAS DE LA SALUD. UNIVERSITAT JAUME I. CASTELLÓN DE LA PLANA.

\title{
AUTOCUIDADOS Y EDUCACIÓN TERAPÉUTICA EN HIPERTENSIÓN. UNA REVISIÓN INTEGRADORA
}

Autores: Balaguer Pallares I, Barrué García P, González Peris R, Llagostera Reverter I, Sanchis Rico P, Aymerich Palomar R; González Chordá VM.

Correo electrónico: al228012@uji.es

Institución: Universitat Jaume I.

Tipo de comunicación: oral.

Objetivo: la educación terapéutica en los pacientes hipertensos debe dirigirse a la modificación de hábitos de vida relacionados con la alimentación, el ejercicio, o la adherencia a las recomendaciones, para conseguir una mejor 
evolución de la enfermedad. El objetivo de este estudio fue revisar la literatura reciente relacionada y conocer las mejores medidas para permitir una elevada tasa de cumplimiento de las recomendaciones.

Material y método: Se realizó una búsqueda bibliográfica en las bases de datos PudMed, Lilacs, Scielo y Enfispo en Marzo de 2013. El límite temporal fue de 5 años. Se buscaron las palabras clave en título, abstract y como descriptores.

Resultados: Se obtuvieron un total de 1050 artículos, de los cuales 978 (93\%) fueron excluidos. El principal motivo de exclusión fue el año de publicación, anterior a 2008. PubMed fue la base de datos con mayor número de artículos, seguida de Lilacs. Los artículos de carácter descriptivo fueron los más predominantes.

Conclusiones: La dieta DASH se ofrece como una posible alternativa para el desarrollo de intervenciones relacionadas con la alimentación en pacientes hipertensos. Respecto al ámbito del ejercicio físico, el programa CIMEQ parece que muestra beneficios en el control de las cifras de TA. No se han encontrado estrategias solidas que garanticen la adherencia a las recomendaciones terapéuticas relacionadas con el ejercicio físico y la alimentación. Parece necesario desarrollar investigaciones de calidad que ayuden a conocer cuáles son las mejores estrategias en la educación terapéutica en pacientes con hipertensión. 